\section{Opinion \\ Climbing Brain Levels of Organisation from Genes to Consciousness}

\author{
Jean-Pierre Changeux ${ }^{1, *}$
}

Given the tremendous complexity of brain organisation, here I propose a strategy that dynamically links stages of brain organisation from genes to consciousness, at four privileged structural levels: genes; transcription factors (TFs)-gene networks; synaptic epigenesis; and long-range connectivity. These structures are viewed as nested and reciprocally inter-regulated, with a hierarchical organisation that proceeds on different timescales during the course of evolution and development. Interlevel bridging mechanisms include intrinsic variation-selection mechanisms, which offer a community of bottom-up and top-down models linking genes to consciousness in a stepwise manner.

\section{Understanding the Human Brain}

Several international research programs aim to advance understanding of the human brain and its functions by using multidisciplinary approaches, including information technologies. Yet, contemporary brain sciences face serious difficulties. For instance, at the experimental level, most in vivo data originate from behavioural, electrophysiological, or brain imaging recordings, with little reference to the molecular level that is so essential for drug design. From a theoretical and modelling perspective, additional difficulties are encountered. For instance, microprocessors are claimed to fall short in representing synaptic and neuronal dynamics [1]; in addition, the use of Bayesian statistics (see Glossary) is criticised in the modelling of cognitive processes [2]. Moreover, modelling studies often disregard the evolutionary and developmental dynamics of brain hierarchical organisation and their underlying molecular mechanism. Thus, there is an urgent need to integrate concepts and data from the disparate and highly individualised brain science disciplines within a unified framework of brain biology. Here, I delineate some of these difficulties and suggest plausible strategies to bridge the divide between the fast-moving extremes of the field: genes at one end, consciousness at the other. The challenge is to link the multiple 'brain pictures' arising from current approaches, not necessarily into a unique model, but into a coherent and open community of brain models.

\section{Nesting Models of the Brain}

Brain network models typically comprise assemblies of interconnected neurons with algorithmically defined function amenable to formal computation [3]. This mode of description faces the complexity of an arrangement of 100 billion neurons that arose over a million years of evolution and almost 15 years of individual postnatal development. A possible strategy is to penetrate the jungle of brain physical organisation and tangentially cleave it into multiple nested levels of structural organisation [4]. The definition of a given level classically relies upon the structural (anatomical) characteristics of its elementary components and the particular functions (or properties) unique to that level, each higher level proceeding from elements of a lower level and serving further integrated functions above. Moreover, the models aimed at representing and/or simulating a process and/or behaviour on the basis of minimal, yet realistic,

\section{Trends}

The proposed approach is to nest the various intertwined structural and functional levels that compose the brain into a coherent and open 'brain models community' covering multiple timescales.

A critical bridging role between the gene and neuronal levels is assigned to regulatory proteins termed 'TFs'.

TFs regulate disparate genes into coherent assemblies.

The impact of the environment on brain synaptogenesis is modelled as activity-dependent selective stabilisation pruning of synapses.

Long-range connectivity, subject to developmental shaping through interactions with the physical, social, and cultural environment, is proposed to form the bridge between neuronal microcircuitry and higher cognitive functions by globally integrating the underlying neural organisations.

A novel allosteric pharmacology of TFs is proposed for neuropsychiatric diseases.

${ }^{1}$ Collège de France and CNRS UMR 3571, Institut Pasteur, Paris, France

${ }^{*}$ Correspondence:

changeux@noos.fr (J.-P. Changeux). 


\section{Trends in Cognitive Sciences}

architectures and activity patterns most often use a single level of organisation. To attempt a type of modelling that spans several levels, as proposed here, is in itself a theoretical position.

The approach presented here, which may be called a 'dynamical nesting of models', assumes that the brain is a nested assembly of functional structures at multiple levels of organisation, from molecules to consciousness, reciprocally inter-regulated and in constant dynamic evolution, which operates intrinsically through variation-selection mechanisms yet on different timescales, from the million years of human ancestry up to the $100 \mathrm{~ms}$ of psychological operations. This generalised 'Darwinian' paradigm [5-11] implies that, at successive levels of organisation, the processes of variability, selection, and amplification occur and establish close inter-relationships between developing and adult brains and the constantly evolving physical, social, and cultural environment. Such interactive processes are expected to leave anatomical and functional traces within the eminently variable architecture of each individual brain. Given that the spectrum of organisation levels is broad, only four have been selected and are discussed at the risk of being incomplete and biased (Figure 1).

In the brain, the macromolecular level has a fundamental role by imposing inescapable physical constraints upon even the highest levels: for instance, the evolution of the species-specific features of the brain is grounded in genes. The dynamics of signal transduction by receptors and ion channels limit the dynamics of information processing by the brain to the speed of sound, while our computers operate at the speed of light. All brain functions, including the highest, are necessarily rooted in the physics and chemistry of their basic macromolecular components. Furthermore, the system of TFs, which underpins cooperative networks of gene

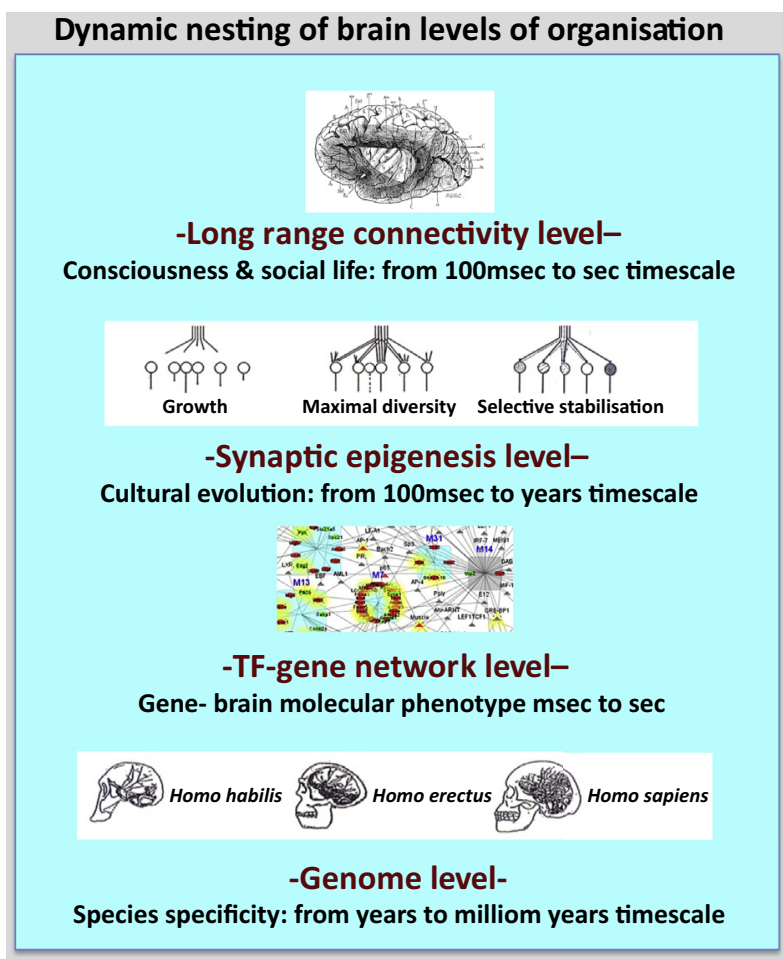

Trends in Cognitive Sciences

Figure 1. Dynamic Nesting of Brain Levels of Organisation. A schematic view of the proposed model of nesting levels of brain organisation and their inter-relationships. As indicated, only a few levels have been selected, on both sides of the neuronal level, but with distinct building blocks and timescale dynamics. For further explanation, see the main text.

\section{Glossary}

Alexia: in pure alexia, individuals have severe reading problems due to cerebral lesions, while other language-related skills, such as naming or writing, remain intact. Allosteric interaction: in contrast to the competitive steric interaction between ligands for a single site, an allosteric interaction occurs between topographically distinct binding sites and is mediated by a conformational change.

Bayesian statistics: a theory in the field of statistics in which the evidence about the true state of the world is expressed in terms of degrees of belief, known as Bayesian probabilities. Bayesian inference is specifically based on the use of Bayesian probabilities to summarise evidence. It is no more than a method of calculus.

Darwinian mechanism: an evolutionary mechanism inspired by the theories of Charles Darwin on the evolution of species but not necessarily occurring at the gene level: for example synapse selection (or neural Darwinism) in the nervous system.

Diffusion tensor imaging tractography (DTI): a magnetic resonance-based neuroimaging technique based upon the observation that parallel bundles of axons and their myelin shield facilitate the diffusion of water molecules along their length, making it possible to visualise white matter tracts in the brain.

Degeneracy: a code that is degenerate is one in which several code words have the same meaning The genetic code is degenerate because there are many instances in which different codons specify the same amino acid. According to the neural Darwinism model, the neural code is degenerate in the sense that different neuronal networks might code the same meaning.

Evolutionary parsimony: the absolute number of structural coding genes in the genome of vertebrates is relatively small $(20000-25000)$ and has not significantly changed during the course of mammalian evolution despite a dramatic increase in brain complexity.

\section{Global neuronal workspace} (GNW): the global neuronal workspace, in which conscious processes are assumed to occur, 


\section{Trends in Cognitive Sciences}

expression, has been favoured and modelled to further decipher the relationship between gene and brain phenotype [12]. The much-studied level of the neuron and its axonal and dendritic processes (together with its cytoskeletal architectures) has been deliberately omitted here except at the synaptic level. Indeed, a fundamental aspect of synaptic evolution, known as synaptic epigenesis, mediates the relationship between the innate dispositions of the brain and its constantly changing environment, and has been formalised $[5,6]$. Lastly, the global network created by the long-range connectivity of the brain has been hypothesised to contribute to the global physical workspace where conscious processes occur [13].

Overall, if any level must be driven by bottom-up rules from the levels underneath, it also depends top down on higher levels via the reciprocal interlevel bridging mechanisms central to this discussion.

\section{Comparative Genomics of the Brain}

Present understanding of the human brain relies on its anatomical evolution, which spanned millions to hundreds of years at the gene level. From mouse to humans, the size of the brain and, in parallel, its total number of neurons, increases from approximately 70 million to 85 billion (plus 50 billion glial cells) $[14,15]$. If the increase in size within the human lineage, from Toumai (Sahelanthropus tchadensis) to Homo sapiens, plausibly reflects that in neuron number [14,15], it does not mean that, at the connectivity level, human brain organisation is simply a full homothetic scale-up of the primate brain. Mammalian brain anatomy dramatically evolved from a brain with 10-20 identified cortical areas in primitive mammals to as many as 180 specialised cortical areas per hemisphere [16] (Figure 2A). Following successive axonal and dendritic branching throughout the brain, especially in the prefrontal cortex (PFC), pathways and synaptic connections greatly increased in number and diversity [17-20]. White matter density rose differentially in parallel $[17,18]$ (Figure 2B,C).

By contrast, the full genome sequences now available for many species (mouse, monkey, chimpanzee, humans, and fossil human ancestors [21-23]) are striking in their relative uniformity (see $[6,7,21,22])$. The haploid genome comprises no more than $20000-25000$ gene coding sequences (only $1.2 \%$ of the human genome) and this number does not vary significantly from mouse to humans. Available comparative genomic data [21-26] unambiguously reveal that the increase of brain anatomical and functional complexity does not reflect a parallel increase in genome complexity, especially at the most recent stages of hominisation.

This may be seen as 'astonishing evolutionary parsimony' [6,7]. A search for the few genetic changes accompanying the emergence of human cognition over the past million years has tentatively identified potential single-gene coding events separating humans from nonhuman primates (see [21-26]), even though many of them might be neutral (for discussion, see [21]). The genes identified are mostly involved in the control of brain growth, neuronal maturation, and neurite outgrowth. For example: (i) brain size: such as the genes associated with microcephaly; (ii) cell division: CDK5 regulatory subunit associated protein 2, growth arrest and DNA-damage-inducible gamma, and Ret finger protein-like 1, 2, and 3; (iii) nerve cell maturation: neuroblastoma break-point family genes. Others are directly associated with neuronal functions, such as solute carrier family 2 (facilitated glucose transporter) member 1, dopamine receptor D5, and glutamate ionotropic receptor NMDA 3A and 3B; and possibly neurite outgrowth, such as SLIT-ROBO Rho GTPase activating protein 2, a gene that is present once in apes but has duplicated three times in humans. Last but not least, some genes have been related to language and speech, such as forkhead box P2 (FOXP2) and protocadherin $11 \mathrm{X}$ - and $\mathrm{Y}$-linked. Inactivation of one copy of FOXP2 in humans results in severe deficits in speech production, yet it encodes a protein that is conserved among mammals (which do not speak) but carries two amino substitutions in humans involves a subset of cortical pyramidal cells with long-range excitatory axons, particularly dense in prefrontal, temporoparietal, and cingulate regions. It forms a horizontal network interconnecting multiple specialised, automatic, and nonconscious processors that broadcast conscious information to many other processors, brain-wide. Hierarchical trees of gene expression patterns: see TF-gene networks below. Ignition: according to the GNW theory, the conscious content of a given sensory input, such as seeing, hearing, and so on, is assumed to be encoded by the sustained and amplified activity, referred to as ignition, of a subset of neurons from the GNW, the rest being inhibited. Interlevel bridging mechanisms: central to the views presented in this article, interlevel bridging mechanisms are essential bottom-up and top-down regulatory processes that span levels of brain organisation and integrate the multiple timescale dynamics of a stratified brain evolution and development from genes to consciousness.

Synaptic epigenesis and synaptic pruning: the theory of the Epigenesis of Neuronal Networks by Selective Stabilisation of Synapses assumes that, at every critical step of development, a given synaptic network is transiently larger and less specified than in the adult and the state of activity of the network, spontaneous or evoked by the outside world, regulates the stabilisation versus. degeneration (pruning) of labile synapses.

TF-gene networks and transcription modules: TFs are able to regulate an ensemble of genes that have common DNA regulatory elements that encode other TFs and control the transcription of their own structural gene, thus generating autocatalytic feedback loops and creating cooperative gene assemblies. These assemblies may even become linked into higher-order ensembles, building up hierarchical trees of gene expression patterns, linking genes together into defined ensembles and creating a higher structural level of organisation above the gene level: the TF-gene network level. A defined TF set of genes encoding a given molecular brain phenotype can be 


\section{Trends in Cognitive Sciences}

(reviewed in [22]). FOXP2 might be related to language processing, but cannot be dubbed the 'language gene'.

Moreover, the important but largely unexplored vast noncoding regions of the human genome, its dark matter, are known to include point mutations, rearrangements, transposable element movements, and other changes [26] that are absent in other mammals. Some regions exhibit accelerated evolution $[27,28]$ together with changes in DNA regulatory sequences [28,29]. These genomic comparisons, and the observation that over 10000 single-gene mutations cause a multitude of severe brain disorders in humans, confirm the strong genetic constraints imposed on the evolution of the human brain and its development. Still, at this stage, the actual few discrete genetic events that caused the fast increase in brain complexity during the past million years of hominisation remain unidentified. It might be that the difficulty faced by such identification arises from the particular relationships between genes and brain phenotypes, which are explored in the next section.

\section{Cooperative Gene Assemblies and TF Networks}

The central dogma of genetics, that one gene encodes one protein itself encoding one phenotype, no longer holds for the brain or, in particular, for its cognitive functions $[6,7]$. referred to as a 'transcription module'.

Transcription factors (TFs): regulatory proteins that bind to a specific DNA regulatory element present in the promoter region, and thereby control (inhibit or stimulate) transcription of the adjacent structural genes into mRNA and subsequently into protein.
(A)

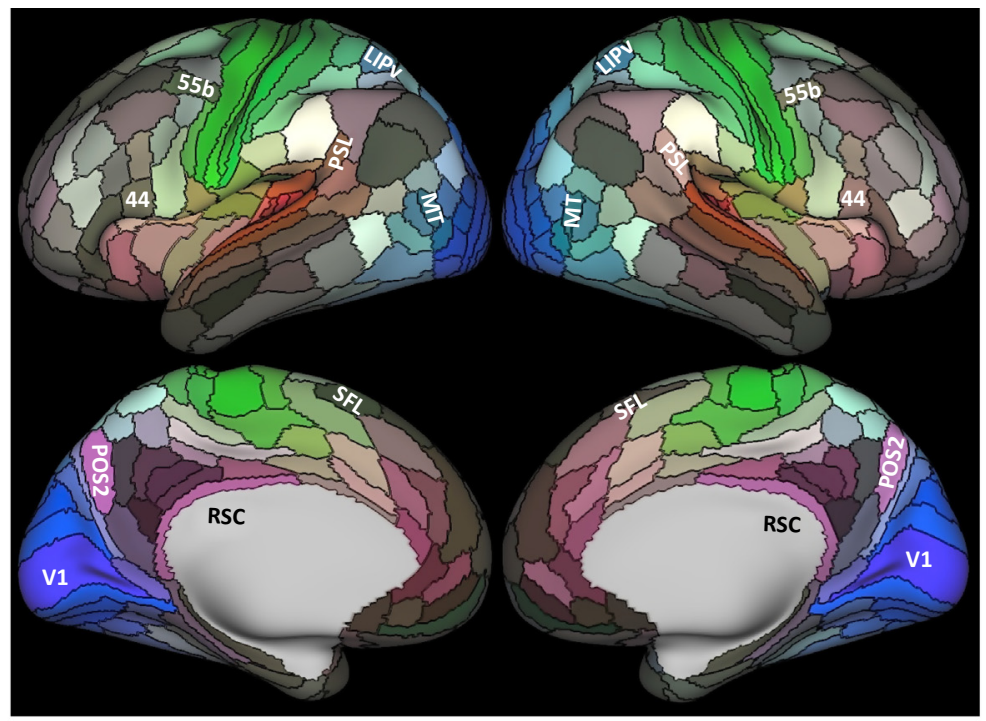

(C) Full-term birth

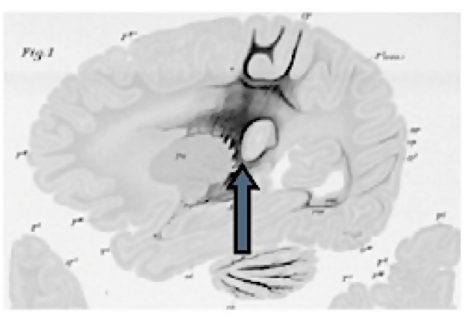

$4 \mathrm{w}$ post term

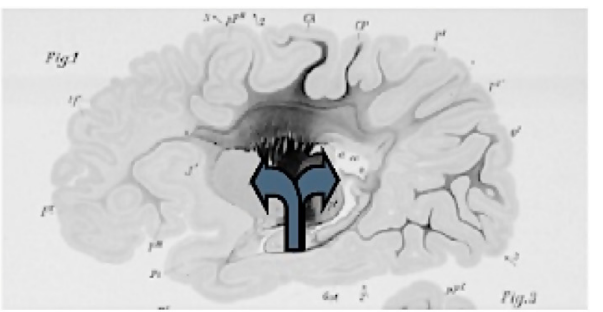

(B)
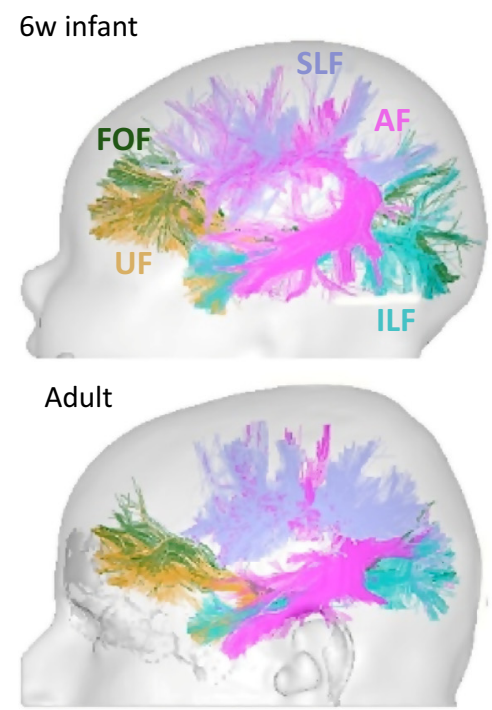

$12 \mathrm{w}$ post term

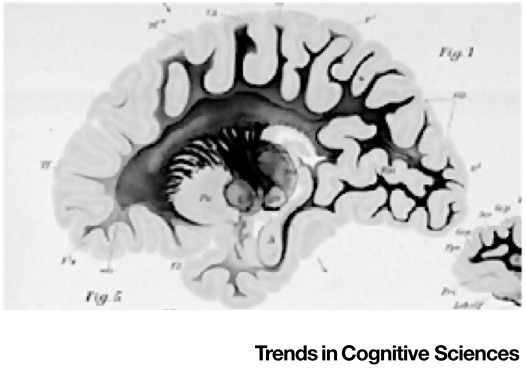

Figure 2. The Human Brain Connectome and Its Development. (A) Neuroimaging data from the Human Connectome Project. They reveal 180 areas on the cerebral cortex delineated and identified in the left or right hemisphere and displayed here on an inflated cortical surface. (B) Development of the long-range association tracts followed by tractography in a 6-week-old infant and in an adult adjusted at the same scale. The image reveals similar global architecture but with significant epigenetic elimination of axonal branches in the adult. (C) White matter maturation followed by myelin stain in postmortem brains illustrating the progression of myelination of tracts during the first trimester after birth. Reproduced from [16] (A) and [113] (B,C). 


\section{Trends in Cognitive Sciences}

Instead, one may posit succinctly that 'gene networks encode neuronal networks' and advocate a radical change in modelling approach. Abundant behavioural genetic studies with simple models systems $[30,31]$, in particular with Drosophila, have revealed that even simple behavioural features most often result from the action of multiple genes and that these genes interact extensively in nonlinear ('epistatic') ways [31].

Since these early studies, a rich body of rodent and human brain gene transcription data has been produced [32-34] and interpreted in terms of networks, where the relationship between genes was specified in the standard formal terms of system biology. Recently [12], a detailed molecular mechanism for cooperative relationships among gene expression data was proposed and modelled, based on an extension of the bacterial operon model [35] to the developmental regulation of eukaryotic gene transcription [36-39]. In the operon scheme, a repressor protein, a TF, binds to a specific DNA element in the promoter region, and thereby controls (inhibits or stimulates) the transcription of adjacent structural genes into mRNA and subsequent protein synthesis (Figure 3). The concept introduced here is that TFs may contribute to a new level of organisation distributed above the gene level.

In eukaryotes, a given DNA element may be present in various promoters within the genome. Since TFs may serve as diffusible signalling proteins, a given TF may bind and, thus, affect populations of genes that share identical (or homologous) promoter elements. In addition, these DNA elements may coexist with others in the promoters, thus creating links between even wider populations of distinct genes. Moreover, TFs often comprise two dissimilar subunits (forming heterodimers), each originating from distinct expression systems, creating additional bridges between pathways. Lastly, TFs also regulate genes encoding other TFs and, thus, control transcription of their own structural gene [38-42], generating autocatalytic feedback loops. Consequently, nonlinear relationships arise among genes, creating co-operative gene assemblies [12]. Even these assemblies (Figure 3C) become linked into higher-order ensembles to form hierarchical trees of gene expression patterns $[14,39,43,44]$ (Figure 3B). Thus, established mechanisms that link genes into defined ensembles create a higher structural level of organisation above the gene level, with its own dynamics and regulation: the TF-gene network level.

Last but not least, since TFs belong to intracellular and intercellular signalling pathways, they may themselves be under stringent metabolic control (by covalent modification, phosphorylation, or ubiquitination) and subserve the spontaneous and/or evoked activity of these neuronal networks [44-46]. In sum, mechanisms exist that form interlevel bridges from the activities, including cognitive ones, of overarching neuronal networks down to the TF network and, hence, gene levels.

\section{The TF-Gene Network Model and Autism Spectrum Disorder}

Actual gene-expression time course data obtained in the developing rat [47] and, less so, in human brain [21-48] (1086 genes with $a \geq 2.5$-fold change) have been analysed in terms of the TF-gene network model [12]. Correlated groups of co-expressed genes were found to differ during development in cerebral cortex, hippocampus, and hypothalamus. In the cerebral cortex, characteristic gene expression patterns were identified. Some were upregulated during prenatal development and concerned early neurogenesis, neuronal differentiation, and migration. Others were upregulated soon after birth, implicated in neurite outgrowth and synapse formation, and affected by the early physical, social, and cultural environment (see the next section). As discussed below, during late postnatal development, the upregulated pathways were those involved in the late synaptic epigenesis of cortical, including long-range connections $[13,22,23]$. The underlying hierarchical tree of TF-gene networks that controls specific expression patterns during cortical development (Figure 3C) [12] was elucidated. Transcription 


\section{Trends in Cognitive Sciences}

(A)

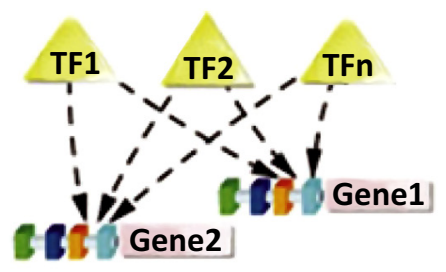

(B)

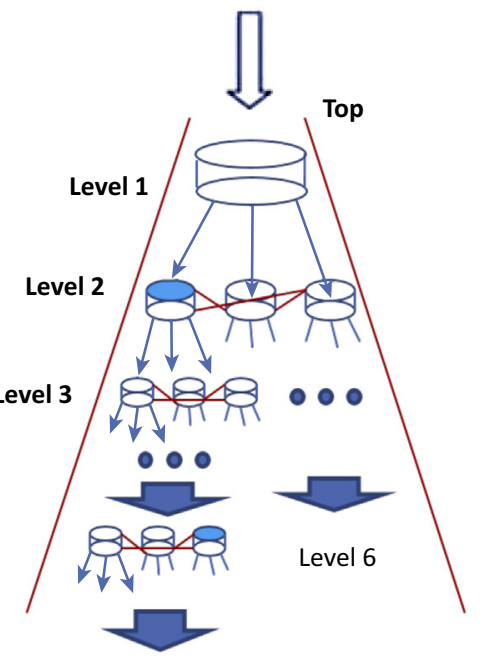

Bottom (c)

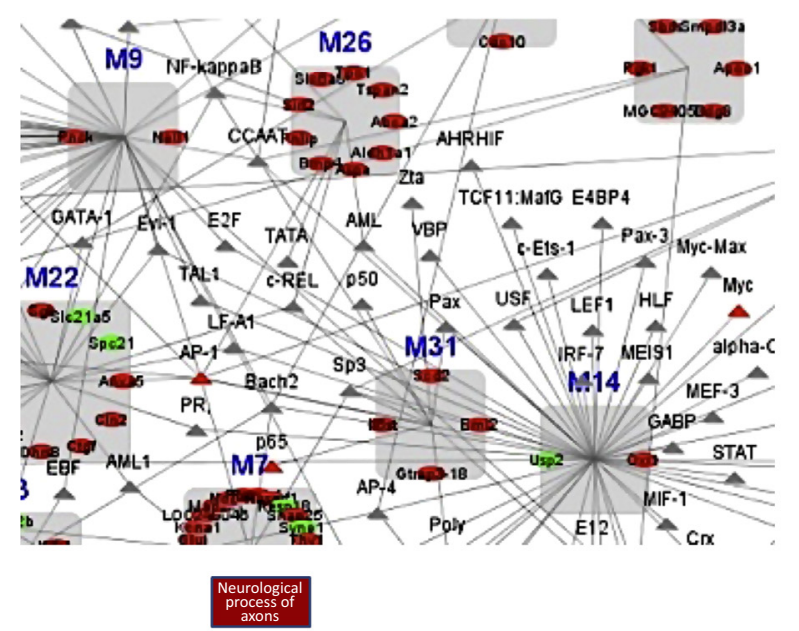

(D)

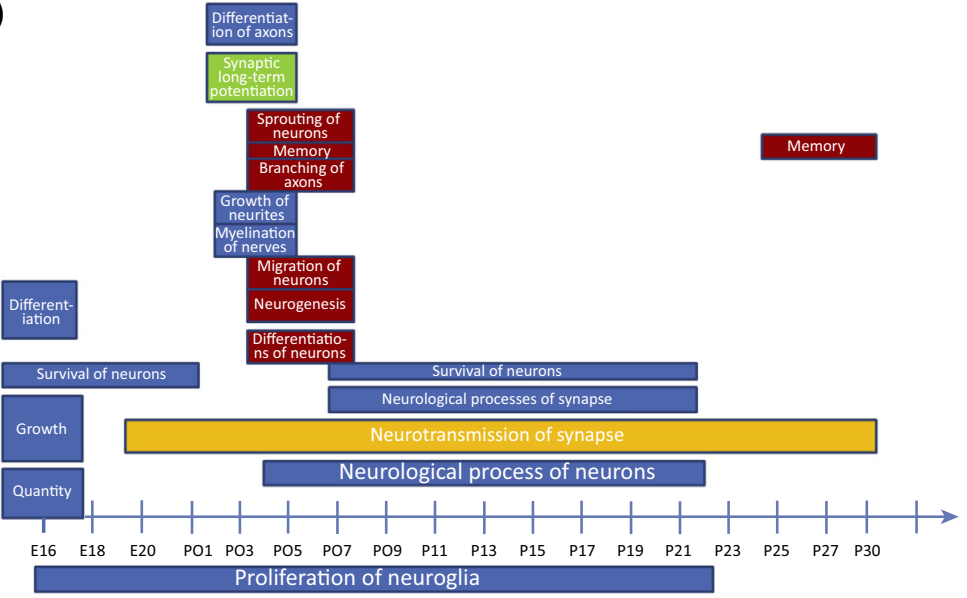

Trends in Cognitive Sciences

Figure 3. Transcription Factor (TF)-Gene Network Model and Development of Gene Expression Patterns in Cerebral Cortex. (A) TFs belong to a class of proteins that control the rate of transcription of DNA genes into mRNA when they bind to specific DNA sequences from a regulatory device called an 'operator'. TFs include the lac repressor in Escherichia coli (refs in [111]) and the nuclear hormone receptors. TFs are homo-(hetero)dimers of two subunits, each comprising an $\mathrm{N}$-terminal domain that interacts with the specific DNA element of the operator, an unstructured linker, the core of the repressor, and the ligand-binding domain. (A) further illustrates how to investigate the formation of TF-gene networks during the early stages of brain development. TF1, TF2, TFn . . are represented by yellow triangles and promoters by lines of coloured dots. Systematic search for co-expressed genes (gene 1, gene 2) and their promoter frameworks results in the identification of a self-organised map representing groups of co-expressed genes. (B) The hierarchical TF-gene network at day 01PN of brain development. (C) A detailed TF-gene network at day PN 30 . TFs are represented by triangles coloured only when they are present in the initial gene set; ovals represent the coherent gene groups (red, upregulated; green, not upregulated) forming a hierarchical coherent gene group model of brain development. (D) Gene expression patterns and relevant neuronal processes obtained from the analysis of rat cortex development. The data were automatically selected using P values for nonrandom participation of the upregulated genes of the cerebral cortex. A major qualitative change starts on days P04-P07 immediately following birth, possibly as a consequence of Lagercrantz's 'stress of being born'. Many of these genes are involved in synaptic epigenesis. Reproduced from [12].

modules [12,49-51] co-regulated by common TFs were found, consistent with recent data [52] on evolutionary changes in promoter and enhancer activity during human corticogenesis (see also $[53,54])$.

Among other predictions, the TF-gene network model exhibits properties of divergence (a given TF affects multiple TFs and gene expression patterns) and convergence (several TFs modulate a common gene expression or phenotype). As a test of the model, the 'risk genes' data for autism spectrum disorders (ASD) (reviewed in [55]) were selected. ASD is an earlyonset neuropsychiatric disorder diagnosed in children as an impairment of social communication. Several laboratories have identified a variety of risk genes, such as the number of genes 


\section{Trends in Cognitive Sciences}

responsible for 'monogenic' forms of ASD likely numbering over 400 [55, see also 56]. These genes generally concern brain development, synaptogenesis, and neuronal plasticity (Figure 3D). The paradox is that, despite the widespread involvement of these genes, their alterations do not systematically cause global brain deficits or intellectual disability, although comorbidities are frequent [57]. All cause a particular cognitive deficit: an alteration of social consciousness. Such an enigmatic relationship is accounted for by the 'convergence' prediction of the TF-gene network model [12]. It means that the numerous risk genes all belong to, and are controlled by, a common hierarchically organised network of TF genes that converge upon the biosynthesis of certain critical neural components (see the following sections). Their mutation creates a common alteration of social behaviour.

Another observation consistent with the TF-gene network model [12] is that the pattern of ASD risk gene expression during development exhibits two maxima, one prenatal, the other soon after birth. This is consistent with the view that ASD is a developmental condition that starts prenatally, further develops postnatally, and reaches its neural and behavioural phenotype by mobilising different sets of genes during each period.

In conclusion, the TF-gene network model accounts for some enigmatic issues raised by recent studies of ASD risk genes, in particular their great diversity despite the fact that they encode a common behavioural phenotype. It may also explain phenotypic variance in the way in which gene patterns are expressed in genetically identical individuals; however, this feature has not yet been examined.

\section{Epigenesis of Neuronal Networks by Selective Synapse Stabilisation}

A most important and unique feature of human brain evolution is the extension of postnatal development for up to 15 years (Figure 2). An approximately fivefold increase in brain weight accompanies this development [58], during which about half of all adult synaptic connections are formed at a very fast pace (approximately 0.5 million synapses per second). This multistep process directly contributes to the formation and shaping of the synaptic architecture of the adult human brain in a close inter-relationship with the physical, social, and cultural environment.

At nested critical periods during the development of the brain, the phenotypic variability of nerve cell distribution and position, as well as the exuberant spread and the multiple transient connectivity configurations resulting from the erratic growth cone wanderings, produce a broad diversity of synaptic connections. This transient diversity is then reduced by selective stabilisation of some of the labile contacts and elimination (or retraction and/or pruning) of others. Such trial-and-error mechanisms that occur within the framework of the speciesspecific genetic blueprint for the basic architecture of the brain, formally resembles the variation-selection process of an evolutionary Darwinian but epigenetic process [5-11], The exuberant outgrowth of synapses would contribute to the variability component, the activitydependant selective stabilisation and/or pruning process to the selection one. A critical implication of the proposed model [5] is that evolution of the connective state of individual synaptic contacts is governed globally, and within a given time window, by the total activity afferent onto the postsynaptic soma. It includes, as a particular case, the standard Hebbian time-coincidence relationship. Activity of the postsynaptic cell in turn regulates, in a retrograde manner, the stabilisation and/or elimination of afferent synapses.

As a consequence, it can be shown mathematically that the same afferent message may stabilise different connective organisations that nevertheless result in the same input-output (behavioural) relationship [5]. Thus, the neuronal connectivity code exhibits degeneracy; that is, different code words (connection patterns) carry the same meaning (function). One 


\section{Trends in Cognitive Sciences}

prediction of the synapse stabilisation theory is that the synaptic connectivity of genetically identical individuals (i.e., monozygotic twins) may display phenotypic variance. This was demonstrated using serial electron microscopy scans of genetically identical individuals (identical twins) of parthenogenetic fish (Poecilia formosa) [59]. The exact branching pattern of an identified motor neuron was shown to vary not only among individuals, but also between left and right in the same animal. More recently. a left-right comparison in rat of interscutularis muscle innervation [60] revealed significant variability of axon branching. Yet, the fish all swim the same way and the left and right limbs of the rat work the same. In addition, parcellation of human cortex in identical twins exhibits significant variability $[61,62]$. The synapse selection model offers a neural example to what philosophers call 'multiple realisability'; that is, the nonunique (degenerate) mapping of a given 'invariant' function to the underlying neural organisation.

The selective stabilisation model accommodates early observations in the visual system [63] and the neuromuscular junction [64]. It has since been abundantly documented as 'synaptic pruning' [65-69] (Box 1). In humans, the total number of synapses in the cortex peaks within the first 3 years of age, then steadily declines to a plateau at around puberty $[70,71]$. The decline observed during late childhood plausibly reflects the underlying rich nesting of selection steps in a cascade of critical periods that proceeds far beyond puberty [63-73]. Detailed investigations of the cellular and molecular mechanisms involved in synapse selection [45,73-76] (Box 1) reveal how the activity of the neuronal network may control, in a top-down manner, the genes involved in synapse selection as the TF-gene networks to which they belong (Figure 3D).

Dynamic evolution of brain synaptogenesis appears essential for the genesis, internalisation, and intergenerational transmission of culture [6,7], as well as for the social communication of individual experience. Among other manifestations of cultural evolution, writing and reading appear as recent inventions (approximately 8000 years ago) that put considerable demands on our cognitive system. Historically, the first evidence for specialised brain circuits for writing and reading was the discovery [77] of pure alexia, which causes severe reading problems, while

Box 1. Cellular and Molecular Mechanisms of Synapse Selection

The cellular and molecular mechanisms involved in synapse selection include GABAergic inhibition, which contributes to the 'opening' of the critical period where synapse selection occurs (reviewed in [73]). A shift in the excitatory-inhibitory balance is associated with the maturation of fast-spiking GABAergic inhibitory neurons that synthesise parvalbumin and are localised in layers III/IV of the cerebral cortex [73]. Pharmacological agents that accelerate GABA circuit function (such as benzodiazepines) elicit precocious onset, whereas genetic manipulations (such as the deletion of genes involved in GABA synthesis) or environmental disruption (such as dark rearing or hearing loss) lead to a delay of the critical period.

Neurotrophic factors, including growth factors (NGF), brain-derived neurotrophic factor (BDNF), neurotrophin-4 (NT-4), and neurotrophin-3 (NT-3), are important regulators of visual cortical plasticity [74]. In transgenic mice in which the postnatal rise in BDNF in the fore-brain was genetically accelerated, a precocious termination of the critical period of ocular dominance plasticity was found, which correlated with an accelerated maturation of GABAergic inhibitory circuitry $[74]$.

In addition, homeoproteins, such as Otx2, have been reported to have a role in synaptic evolution during critical periods of development [45]. For instance, Ot×2 accumulates in an activity-dependant manner inside the fast-spiking GABAergic neurons. Intriguingly, Otx2 is not expressed by these cells but instead imported from one or several external sources. Therefore, it was proposed that, in the mouse, Otx2 accumulation by fast spiking neurons cells is necessary and sufficient for a binocular critical period opening at 20 days after birth and closing at 40 days after birth [45].

Postnatal differential activation, en cascade, of the synaptome genes examined ([12], but see [21]) coincides with the postnatal epigenesis of cortical synaptic networks, including neurite growth and branching, synapse formation, synaptic transmission (neurotransmission) genes and, last but not least, the genes involved in the selective stabilisation of synapses, such as the ubiquitin-proteasome system [76] [see main text and Figure 3 (main text)]. 


\section{Trends in Cognitive Sciences}

other language-related skills, including naming, oral repetition, auditory comprehension, or writing, remain intact. Alexia results from circumscribed brain lesions that include the supramarginal and angular gyri, which affect specific bundles of myelinated axons. Acquisition of reading and writing may be viewed as an example of epigenetically laid down 'cultural circuits' following epigenetic 'appropriation' of fast-developing connections at around 5 years of age $[78,79]$. It operates at a time of still rapid synaptogenesis and persists into adulthood [79,80]. Written language learning is but one of many social and cultural imprints acquired during the development of the human brain [5-10]. Thus, the adult human brain connectivity may be viewed as a complex intertwining of social and cultural circuits epigenetically laid down during development within the framework of a human-specific genetic envelope. Important 'bridges' could then be established between gene-TF networks and the social and cultural environment.

\section{The Global Neuronal Workspace}

The ultimate level among higher brain functions is that of conscious processing and its diverse modalities. Considerable effort has been spent unravelling its neuronal basis [13,81-87], generating theories, some of which favour global integration (Integrated information theory) [84], whereas others favour specialised neuronal architectures [16,85-87]. Here, I focus on one of the latter: the global neuronal workspace (GNW) [13,86,87], which offers a simple, but possibly useful, hypothetical scheme that adequately fits the multilevel nesting modelling approach (Figure 4A). Among the innumerable neuronal structures available in the brain, the theory privileges a subset of cortical pyramidal cells with long-range excitatory axons, particularly dense in prefrontal, temporoparietal, and cingulate regions, that, together with the relevant thalamocortical loops, forms a horizontal 'neuronal workspace' interconnecting multiple specialised, automatic, and nonconscious processors [13,87]. The conscious content of a given sensory input (seeing, hearing, etc.) is assumed to be encoded by the sustained and amplified activity (termed 'ignition') of a subset of neurons from the GNW, the rest being inhibited. The long-distance axons of these elements then broadcast the information to many other processors, brain-wide and with defined spatial distribution, ultimately leading to a report of conscious experience. In its original formulation, the GNW $[13,87]$ was designed to simulate effortful cognitive tasks and included reward mechanisms as a critical component. It was then successfully applied to fit data from simpler tasks, including masking tasks [86,87].

Recently, social relationships, which, as mentioned, appear altered in ASD, have been introduced into the neuronal architectures for conscious processing $[55,88]$. Social consciousness is assumed to engage cortical areas, including the superior-temporal-sulcus, the temporoparietal junction, and the medial PFC, mostly in the right hemisphere (refs in [88]). Concomitantly, a self-referential core of changing conscious experiences has been related through PET, fMRI, and MEG studies (refs in $[89,90]$ ) to the mobilisation of a paralimbic network of medial PFC/ anterior cingulate and medial parietal/posterior cingulate cortices [see also 97,98]. Interestingly, although still a hypothesis, these views appear consistent with the GNW theory and its simultaneous 'ignition' of a common set of brain regions in conscious processing $[86,87]$.

A common and critical anatomical feature shared by all these systems is the contribution of long-range connections, in particular those involved in top-down reafference [13,91-93], assumed to provide a structural basis for the global experience of being conscious (Figures 2B,C and 4). Among the cortical connections established during pre- and postnatal life are the long-range tracts between the frontal areas ([92-95] and other (including sensory) cortical areas that constitute the GNW $[74,99,100]$. Developmental regulation of these long-range connections may be related, at least by simulation, to the underlying TF-gene networks computational model [12] (Figure 3). Attempts are underway to sort out the particular coherent gene groups and TFs selectively involved in establishing the long-range GNW networks and their myelination at successive postnatal maturation stages, together with the synaptic selection processes 


\section{Trends in Cognitive Sciences}

(A)

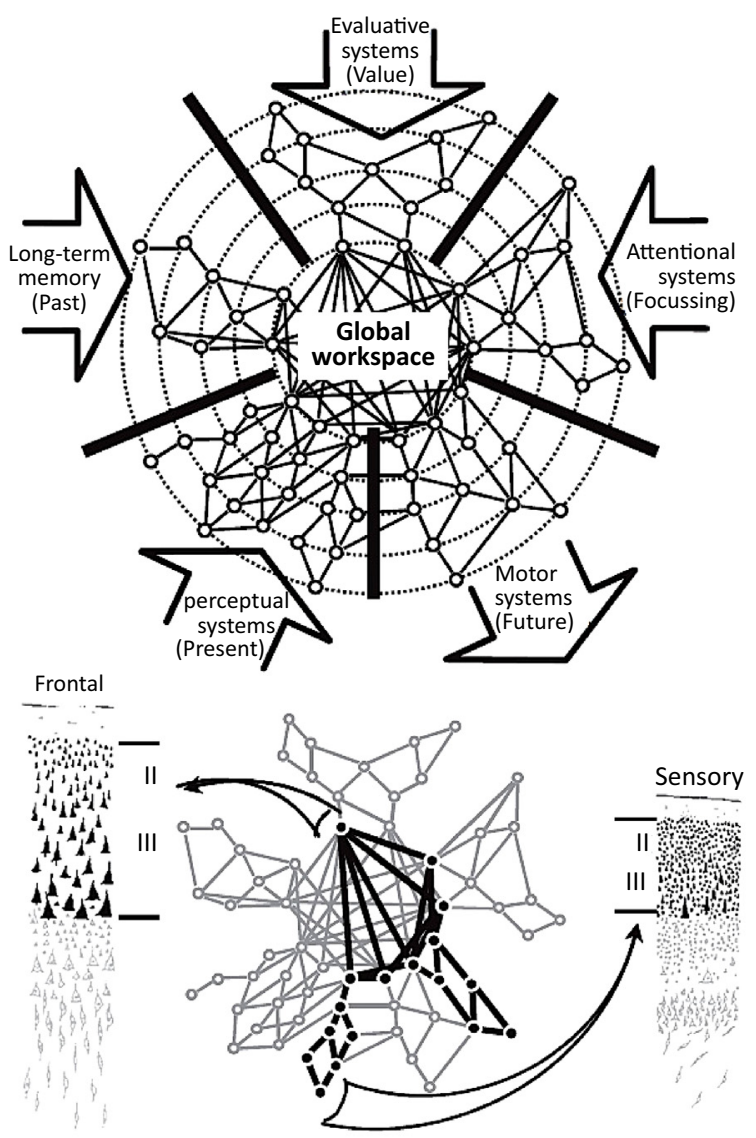

(B)

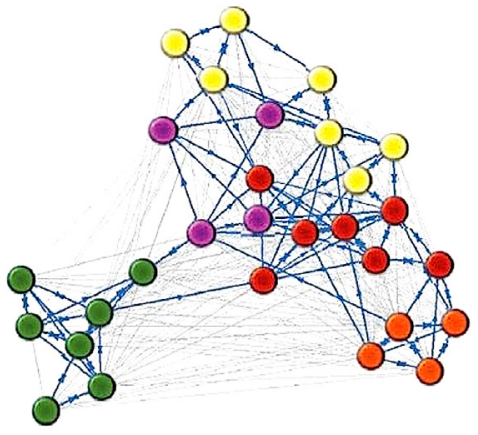

Occipital $\bigcirc$ Parietal $\bigcirc$ Temporal $\bigcirc$ Frontal Prefrontal

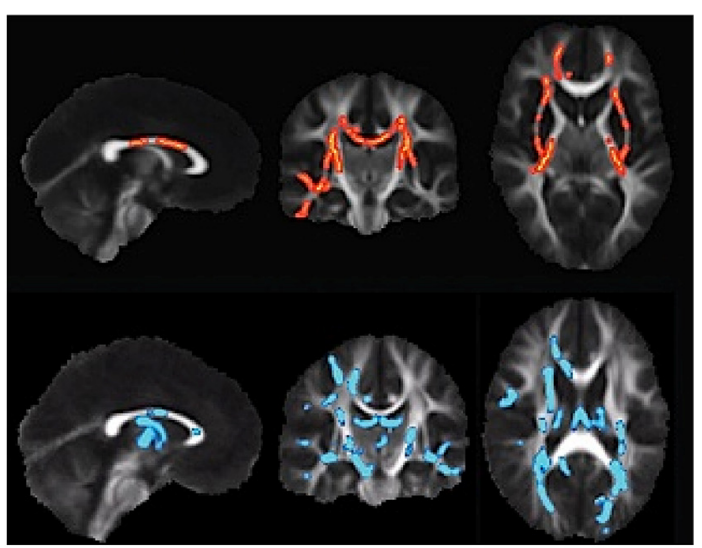

Trends in Cognitive Sciences

Figure 4. The Global Neuronal Workspace and Autism Spectrum Disorder (ASD) Phenotype. (A) Schematic representation of the global neuronal workspace (GNW) model. The model posits that associative, perceptual, motor, attention, memory, and value areas are interconnected to form a higher-level unified workspace where information is broadly shared and broadcasted back to lower-level processors. The GNW connectivity comprises long-distance corticocortical axons from layer II/III large pyramidal cells, which are particularly dense in prefrontal cortex, establishing a prefrontal-parietotemporal-cingular network. (B) Spatial network model of the cerebral cortex connectivity using graph theory. The image shows interactions among cortical areas that underlie higher sensory, motor, and cognitive functions. This image represents a subgraph (using a force-based layout algorithm) considering the $24 \%$ strongest links only (blue arrows). Here, the areas are clustered into functional regions. In agreement with the GNW, the prefrontal-frontal-parietotemporal territories cluster together. (C) Tracking the long projections of neurons to unravel autism. Brain imaging by diffusion MRI. Patients with a deletion of the autism-linked region 16p11.2 (top) have stronger neuronal connections (red) compared with controls, whereas those with a duplication of the region (bottom) show the opposite pattern (blue). Reproduced from [13] (A), [96] (B), and [114] (C).

controlled by spontaneous and evoked activity (see Figure 4 and [12]). These views are also consistent with the above-mentioned proposal that frontal white matter has a critical role in the evolution of human brain size and organisation [17-19] (Figure 2B,C).

Again, the well-documented genetic investigations with patients with ASD offer an exceptional opportunity to test these views. Brain imaging studies (in particular, by diffusion tensor imaging tractography) reveal in some of the patients with ASD studied, a loss or overproduction of long-range axonal fibers in some privileged cortex areas, accompanied by intact or even excessive short-range connectivity [100-112] (Figure 4C). This selective alteration of longrange connectivity, which also varies among individuals [109], might be attributed to altered synapse selection $[12,55,100]$ elicited by some predisposition genes at critical developmental stages (3-5 years) $[60,109]$ (Figure 4C). Lastly, in children with ASD, deficits are noted in the performance of psychophysical tasks dedicated to conscious access [101-105] and even selfconsciousness [101] (also in patients with schizophrenia [61]) suggesting an alteration of the 


\section{Trends in Cognitive Sciences}

GNW [87], which matches the proposal that the common behavioural phenotype of ASD is an alteration of some of the 'highest levels' of consciousness [105,109].

More generally, the ASD example illustrates the concept of interlevel bridging mechanisms operating between high-level cognitive functions, implemented here by a defined set of longrange connections, and the particular TF-gene network concerned. Moreover, the activity state of this long-range network would have a central role in the bridging process by controlling its epigenesis at critical stages of development; that is, at age 2-5 years in children with ASD.

This TF-gene network modelling approach suggests new drug design strategies (Figures 3 and 5). The new molecules should target a given TF within the hierarchical network to block (or enhance) its activity. The compounds may preferentially bind allosteric sites topologically distinct from the TF DNA-binding site [111] (also see allosteric interaction; Figure 5B). These drug candidates might be used to identify the 'gene expression modules' encoding the relevant structural elements that give access to the successive developmental levels of cognitive functions. Also, these compounds could be tested on mouse models that carry mutations homologous to those identified in patients with ASD and display characteristic neural and behavioural phenotypes [55] together with induced pluripotent stem cells. Such a strategy may eventually be applied to other devastating brain diseases.

(A)

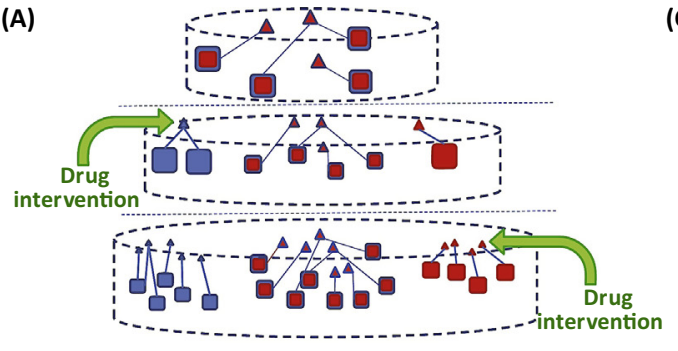

(C)

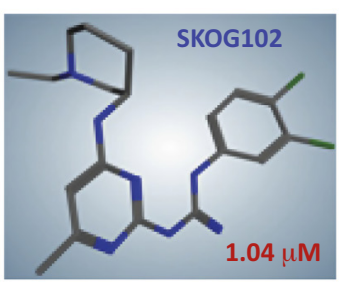

(B)

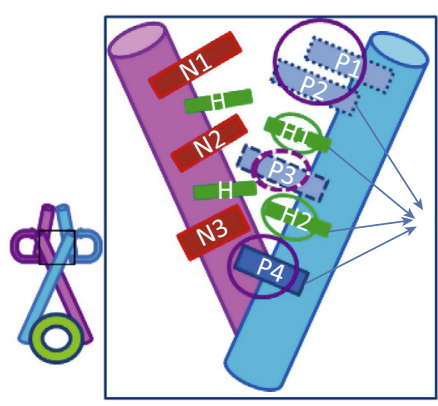

(D)

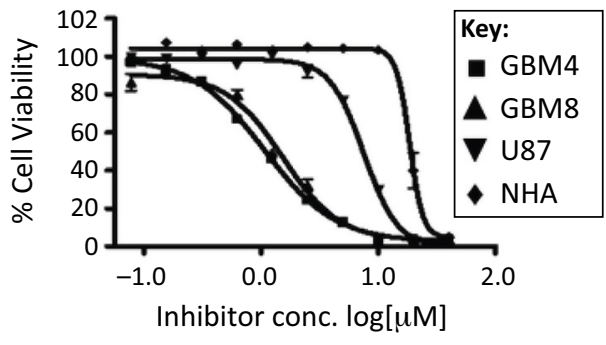

Trends in Cognitive Sciences

Figure 5. An Allosteric Pharmacology of Transcription Factors. (A) Diagram illustrating the hierarchy of transcription factors (TFs) and coherent gene group networks according to [12]: red, autism-related coherent gene groups and TFs; blue, schizophrenia-related coherent gene groups and TFs. The novel strategy of drug design is based on targeting of the drug candidate at a selected TF of the hierarchy. (B) TFs are essentially dimers: the heterodimer shown here comprises two subunit (red and blue). Since most TFs do not have a well-defined ligand-binding site, the strategy [112] is to design allosteric ligands (refs in [111]) that inhibit or enhance the assembly of the subunits into a functional oligomeric dimer (here OLIG2-E47). (C) Formula of the drug candidate SKOG102, one of the selected OLIG2 blockers. (D) SKOG102 blocks the growth of several lines of mouse glioma stem cells, providing a strong proof of principle for the success of the strategy. With the aim of developing treatments against autism spectrum disorders (ASD), these strategies can be experimentally tested, first on mouse models, which carry mutations homologous to those identified in patients with ASD and display characteristic neural and behavioural phenotypes (60). If successful, such drugs may interfere with, and even restore, the pathological evolution of mutated TFs in genetically predisposed patients when administered at relevant sensitive stages of brain development and of course under adequate ethical conditions. Reproduced from [12] (A) and [115] (B-D). 


\section{Trends in Cognitive Sciences}

\section{Concluding Remarks and Future Perspectives}

The strategy of 'dynamic nesting of models' has been tentatively explored at four selected levels of brain organisation that involve genes, the TF-gene network, epigenetic action on synapse formation, and the long-range connectivity domain. Obviously, others might be needed, together with additional elementary components (miRNAs, chromatin 'epigenetic' modifications, subcellular structures, such as microtubules). Yet, it may pave the way to new IT modelling approaches that integrate the multiple timescale dynamics of a stratified brain development. In particular, the approach points to original interlevel 'bridging processes' that operate both bottom up and top down, thereby offering concrete mechanisms for top-down causation. By introducing the TF-gene network, the nesting strategy offers original insights into long-distance relationships between genes and cognitive functions. These include the continuous intertwining of brain development and its sociocultural environment, and the relevant phenotypic variance of individual brain connectivity. The suggested approach tentatively illustrates how these relationships are altered in brain disorders such as ASD and proposes, as a corollary, a new strategy for drug design based on the targeting of TFs during development. Lastly, opening future vistas, the attempt to establish a coherent and open community of brain dynamic models might help unify the diverse multidisciplinary approaches aimed at grasping the singular organisation of the human brain (see Outstanding Questions).

\section{Acknowledgements}

I thank Igor Tsigelny for stimulating discussions and cooperation over the past few years, Paul De Weer for careful editing of the papers, and Guillaume Dumas, Jean-Jacques Slotine, Thomas Bourgeron, and Henri Korn for suggestions and comments. This paper received funding from the European Union's Horizon 2020 research and innovation programme under grant agreement No 720270 (HBP SGA1) and SCR 130008 0; 1 UE 64102 (HBP ramp up). It was written, in part, at the Kavli Brain and Mind institute UCSD and Woods Hole Marine Laboratory.

\section{References}

1. Jonas, E. and Kording, K. (2015) Automatic discovery of cell types and microcircuitry from neural connectomics. Elife 4, e04250

2. Sanborn, A.N. and Chater, N. (2016) Bayesian brains without probabilities. Trends Cogn. Sci. 20, 883-893

3. Marr, D. (1982) Vision: A Computational Investigation into the Human Representation and Processing of Visual Information, Freeman

4. Campbell, D. et al. (1974) 'Downward causation' in hierarchically organised biological systems. In Studies in the Philosophy of Biology (Ayala, F.J., ed.), pp. 179-186, MacMillan

5. Changeux, J.-P. et al. (1973) A theory of the epigenesis of neuronal networks by selective stabilization of synapses. Proc. Natl. Acad. Sci. U. S. A. 70, 2974-2978

6. Changeux, J.-P. (1983) L'Homme Neuronal, Fayard

7. Morange, M. et al. (eds.) (2016) L'Homme Neuronal 30 ans après,Dialogue avec Jean-PierreChangeux, Rue d'Ulm.

8. Edelman, G. (1987) Neural Darwinism: The Theory of Neuronal Group Selection, Basic Books

9. Edelman, G. (2006) Second Nature: Brain Science and Human Knowledge, Yale University Press

10. Barkow, J. (ed.) (1992) The Adapted Mind: Evolutionary Psychology and the Generation of Culture, Oxford University Press

11. Campbell, J.O. (2016) Universal Darwinism as a process of Bayesian inference. Front. Syst. Neurosci. 10, 49

12. Tsigelny, I.F. et al. (2013) A hierarchical coherent-gene-group model for brain development. Genes Brain Behav. 12, 147-165

13. Dehaene, S. et al. (1998) A neuronal model of a global workspace in effortful cognitive tasks. Proc. Natl. Acad. Sci. U. S. A. 95, 14529-14534

14. Herculano-Houzel, S. (2012) The remarkable, yet not extraordinary, human brain as a scaled-up primate brain and its associated cost. Proc. Natl. Sci. Acad. U. S. A. 109 (Suppl. 1), $10661-$ 10668
15. Gabi, M. et al. (2016) No relative expansion of the number of prefrontal neurons in primate and human evolution. Proc. Natl. Acad. Sci. U. S. A. 113, 9617-9622

16. Glasser, M.F. et al. (2016) The Human Connectome Project's neuroimaging approach. Nat. Neurosci. 19, 1175-1187

17. Semendeferi, K. et al. (2002) Humans and great apes share a large frontal cortex. Nat. Neurosci. 5, 272-276

18. Smaers, J.B. et al. (2010) Frontal white matter volume is asso ciated with brain enlargement and higher structural connectivity in anthropoid primates. PLoS One 5, e9123

19. Elston, G.N. et al. (2011) Pyramidal cells in prefrontal cortex of primates: marked differences in neuronal structure among species. Front. Neuroanat. 5, 2

20. Liu, X. et al. (2012) Extension of cortical synaptic development distinguishes humans from chimpanzees and macaques.

21. Somel, M. et al. (2013) Human brain evolution: transcripts, metabolites and their regulators. Nat. Rev. Neurosci. 14 112-127

22. Paabo, S. (2013) The human condition - a molecular approach? Cell 157, 216-226

23. Geschwind, D.H. and Rakic, P. (2013) Cortical evolution: judge

24. Langergraber, K.E. et al. (2012) Generation times in wild chimpanzees and gorillas suggest earlier divergence times in great ape and human evolution. Proc. Natl. Acad. Sci. U. S. A. 109 15716-15721

25. Vallender, E.J. et al. (2006) Genetic basis of human brain evoution. Trends Neurosci. 31, 637-644

26. Chimpanzee Sequencing and Analysis Consortium (2005) Initial sequence of the chimpanzee genome and comparison with the human genome. Nature 437, 69-87

27. McLean (2011) Human-specific loss of regulatory DNA and the evolution of human-specific traits. Nature 471, 216-219 Genome Res. 22, 611-622 the brain by its cover. Neuron 80, 633-647

\section{Outstanding Questions}

How can we better subject current neuronal models of cognitive functions to the strong bottom-up and top-down constraints of the molecular level?

Why not attempt to identify additiona levels of brain organisation and the relevant interlevel bridging processes?

Would the role of TF networks in mediating the gene expression patterns that produce the cognitive phenotype be a fertile field of future investigation?

How can we search for novel interlevel 'bridging' processes and methods?

Is synaptic epigenesis of brain connectivity the sole critical link between the sociocultural environment and postnatal development?

How can we investigate neuropsychiatric disorders under conditions that engage several levels of brain organisation and bridge multiple processes between the gene level and the conscious socioenvironmental one?

How could we convince more neurophysiologists that conscious processing and its pharmacology is an active field of empirical study that can reveal the links between subjective phenomena and objective neurobiological processes?

How can we deal with the important ethical issues raised by this modelling approach, which concern quality of life in the human community? 


\section{Trends in Cognitive Sciences}

28. Holloway, A.K. (2016) Accelerated evolution of enhancer hotspots in the mammal ancestor. Mol. Biol. Evol. 33, 1008-1018

29. Weyer, S. and Pääbo, S. (2016) Functional analyses of transcription factor binding sites that differ between present-day and archaic humans. Mol. Biol. Evol. 33, 316-322

30. Plomin, R. (2013) Behavioral Genetics. (6th edn), Worth Publishers

31. Greenspan, R.J. (2009) Selection, gene interaction, and flexible gene networks. Cold Spring Harb. Symp. Quant. Biol. 74, $131-138$

32. Konopka, G. et al. (2012) Human-specific transcriptional networks in the brain. Neuron 75, 601-617

33. Parikshak, N.N. et al. (2015) Systems biology and gene networks in neurodevelopmental and neurodegenerative disorders. Nat. Rev. Genet. 16, 441-458

34. Johnson, M.R. et al. (2016) Systems genetics identifies a convergent gene network for cognition and neurodevelopmental disease. Nat. Neurosci. 19, 223-232

35. Monod, J. and Jacob, F. (1961) Teleonomic mechanisms in cellular metabolism, growth, and differentiation. Cold Spring Harb. Symp. Quant. Biol. 26, 389-401

36. Britten, R.J. and Davidson, E.H. (1969) Gene regulation for higher cells: a theory. Science 165, 349-357

37. Driever, W. and Nüsslein-Volhard, C. (1998) A gradient of bicoid protein in Drosophila embryos. Cell 54, 83-93

38. Kerszberg, M. and Changeux, J.-P. (1994) A model for reading morphogenetic gradients: autocatalysis and competition at the gene level. Proc. Natl. Acad. Sci. U. S. A. 91, 5823-5827

39. Kerszberg, M. and Changeux, J.-P. (1998) A simple molecular model of neurulation. Bioessays 20, 758-770

40. Noll, M. (1993) Evolution and role of Pax genes. Curr. Opin. Genet. Dev. 3, 595-605

41. Thayer, M.J. (1989) Positive autoregulation of the myogenic determination gene MyoD1. Cell 58, 241-248

42. Walther, C. and Gruss, P. (1991) Pax-6, a murine paired box gene, is expressed in the developing CNS. Development 113, 1435-1449

43. Davidson, E.H. (2010) Emerging properties of animal gene regulatory networks. Nature 468, 911-920

44. Larson, E.B. et al. (2010) Striatal regulation of FosB, FosB, and cFos during cocaine self-administration and withdrawal. J. Neurochem. 115, 112-122

45. Prochiantz, A. and Di Nardo, A.A. (2015) Homeoprotein signaling in the developing and adult nervous system. Neuron 85 , 911-925

46. Kerszberg, M. and Wolpert, L. (2007) Specifying positional information in the embryo: looking beyond morphogens. Cell 130, 205-209

47. Stead, J.D. et al. (2006) Transcriptional profiling of the developing rat brain reveals that the most dramatic regional differentiation in gene expression occurs postpartum. J. Neurosci. 26, 345-353

48. Somel, M. (2010) MicroRNA, mRNA, and protein expression link development and aging in human and macaque brain. Genome Res. 20, 1207-1218

49. Fessele, S. (2002) Regulatory context is a crucial part of gene function. Trends Genet. 18, 60-63

50. Segal, E. et al. (2003) Module networks: identifying regulatory modules and their condition specific regulators from gene expression data. Nat. Genet. 34, 166-176

51. Baitaluk, M. et al. (2012) IntegromeDB: an integral system and biological search engine. BMC Genomics 13, 35

52. Reilly, S.K. (2015) Evolutionary genomics. Evolutionary changes in promoter and enhancer activity during human corticogenesis. Science 347, 1155-1159

53. Emera, D. et al. (2016) Origin and evolution of developmental enhancers in the mammalian neocortex. Proc. Natl. Acad. Sci. U. S. A. 113, E2617-E2626

54. Kirsch, L. and Chechik, G. (2016) On expression patterns and developmental origin of human brain regions. PLoS Comput. Biol. 12, e1005064
55. Bourgeron, T. (2015) From the genetic architecture to synaptic plasticity in autism spectrum disorder. Nat. Rev. Neurosci. 16 551-563

56. Insel, T.R. (2010) Rethinking schizophrenia. Nature 468 187-193

57. Bakken, I.J. et al. (2016) Comorbidities treated in primary care in children with chronic fatigue syndrome/myalgic encephalomyelitis: a nationwide registry linkage study from Norway. BMC Fam. Pract. 17, 128

58. Lagercrantz, H. et al. (2010) The Newborn Brain. (2nd edn) University Press, (Cambridge)

59. Levinthal, F. et al. (1976) Anatomy and development of identified cells in isogenic organisms. Cold Spring Harb. Symp. Quant. Biol. 40, 321-331

60. Lu, J. et al. (2009) The interscutularis muscle connectome. PLoS Biol. 7, e32

61. Steinmetz, H. et al. (1995) Brain (A) symmetry in monozygotic twins. Cereb. Cortex 5, 296-300

62. White, T. et al. (2002) Brain volumes and surface morphology in monozygotic twins cereb. Cortex 12, 486-493

63. Wiesel, T.N. and Hubel, D.H. (1963) Effects of visual deprivation on morphology and physiology of cells in the cats lateral geniculate body. J. Neurophysiol. 26, 978-993

64. Redfern, P.A. (1970) Neuromuscular transmission in new-born rats. J. Physiol. 209, 701-709

65. Benoit, P. and Changeux, J.P. (1978) Consequences of blocking the nerve with a local anaesthetic on the evolution of multiinnervation at the regenerating neuromuscular junction of the rat. Brain Res. 149, 89-96

66. Stretavan, D.W. et al. (1988) Modification of retinal ganglion cell axon morphology by prenatal infusion of tetrodotoxin. Nature 336, 468-471

67. Purves, D. and Lichtman, J.W. (1980) Elimination of synapses in the developing nervous system. Science 210, 153-157

68. Luo, L. and O'Leary, D.D. (2005) Axon retraction and degeneration in development and disease. Annu. Rev. Neurosci. 28 $127-156$

69. Collin, G. and van den Heuvel, M.P. (2013) The ontogeny of the human connectome: development and dynamic changes of brain connectivity across the life span. Neuroscientist 19 , 616-628

70. Huttenlocher, P.R. and Dabholkar, A.S. (1997) Regional differences in synaptogenesis in human cerebral cortex. J. Comp. Neurol. 387, 167-178

71. Bourgeois, J.P. (1997) Synaptogenesis, heterochrony and epigenesis in the mammalian neocortex. Acta Paediatr. Suppl. 422 27-33

72. Petanjek, Z. et al. (2011) Extraordinary neoteny of synaptic spines in the human prefrontal cortex. Proc. Natl. Acad. Sci. U. S. A. $108,13281-13286$

73. Werker, J.F. and Hensch, T.K. (2015) Critical periods in speech perception: new directions. Annu. Rev. Psychol. 66, 173-196

74. Huang, Z.J. et al. (1999) BDNF regulates the maturation of inhibition and the critical period of plasticity in mouse visual cortex. Cell 98, 739-755

75. Choquet, D. and Triller, A. (2013) The dynamic synapse. Neuron 80, 691-703

76. Mabb, A.M. and Ehlers, M.D. (2010) Ubiquitination in postsynaptic function and plasticity. Annu. Rev. Cell Dev. Biol. 26, 179210

77. Dejerine, J.J. and Dejerine-Klumpke, A.M. (1901) Anatomie des Centres Nerveux, Rueff Paris

78. Castro-Caldas, A. (1998) The illiterate brain. Learning to read and write during childhood influences the functional organization of the adult brain. Brain 121, 1053-1063

79. Dehaene, S. et al. (2010) How learning to read changes the cortical networks for vision and language. Science 330, 13591364

80. Szwed, M. et al. (2014) Effects of literacy in early visual and occipitotemporal areas of Chinese and French readers. J. Cogn. Neurosci. 26, 459-475 


\section{Trends in Cognitive Sciences}

81. Crick, F. and Koch, C. (2003) A framework for consciousness. Nat. Neurosci. 6, 119-126

82. Block, N. et al. (2014) Consciousness science: real progress and lingering misconceptions. Trends Cogn. Sci. 18, 556-557

83. Hameroff, S. (2006) Consciousness, neurobiology and quantum mechanics. In The Emerging Physics of Consciousness (Tuszynski, J.A., ed.), pp. 192-251, Springer Science \& Business Media

84. Tononi, G. and Edelman, G.M. (1998) Consciousness and complexity. Science 282, 1846-1851

85. Jasper, H.H. (1977) Wilder penfield: his legacy to neurology. The centrencephalic system. Can. Med. Assoc. J. 116, 1371-1372

86. Dehaene, S. and Changeux, J.P. (2005) Ongoing spontaneous activity controls access to consciousness: a neuronal model for inattentional blindness. PLoS Biol. 3, e141

87. Dehaene, S. and Changeux, J.P. (2011) Experimental and theoretical approaches to conscious processing. Neuron 70, 200-227

88. Graziano, M.S. and Kastner, S. (2011) Human consciousness and its relationship to social neuroscience: a novel hypothesis. Cogn. Neurosci. 2, 98-113

89. Lou, H.C. et al. (2016) Towards a cognitive neuroscience of selfawareness. Neurosci. Biobehav. Rev. Published online April 11, 2016. http://dx.doi.org/10.1016/j.neubiorev.2016.04.004

90. Posner, M.I. et al. (2007) The anterior cingulate gyrus and the mechanism of self-regulation. Cogn. Affect. Behav. Neurosci. 7 , 391-395

91. von Holst, E. and Mittelstaedt, H. (1950) The reafference principle. Interaction between the central nervous system and the periphery. In Selected Papers of Erich von Holst: The Behavioural Physiology of Animals and Man, pp. 39-73, Methuen

92. Buschman, T.J. and Miller, E.K. (2014) Goal-direction and topdown control. Philos. Trans. R. Soc. Lond. B Biol. Sci. 369, 20130471

93. Koechlin, E. (2016) Prefrontal executive function and adaptive behavior in complex environments. Curr. Opin. Neurobiol. 37, 1-6

94. Fuster, J. (2015) The Prefrontal Cortex. (5th edn), Academic Press

95. Goldman-Rakic, P.S. (1999) The physiological approach: functional architecture of working memory and disordered cognition in schizophrenia. Biol. Psychiatry 46, 650-661

96. Markov, N.T. et al. (2013) Cortical high-density counterstream architectures. Science 342, 1238406

97. Rømer Thomsen, K. (2013) Altered paralimbic interaction in behavioral addiction. Proc. Natl. Acad. Sci. U. S. A. 110, 4744-4749

98. Tang, Y.Y. et al. (2015) Circuitry of self-control and its role in reducing addiction. Trends Cogn. Sci. 19, 439-444
99. Changeux, J.P. and Lou, H.C. (2011) Emergent pharmacology of conscious experience: new perspectives in substance addiction. FASEB J. 25, 2098-2108

100. Belmonte, M.K. et al. (2004) Autism and abnormal development of brain connectivity. J. Neurosci. 24, 9228-9231

101. Toichi, M. et al. (2002) A lack of self-consciousness in autism. Am. J. Psychiatry 159, 1422-1424

102. Ben Shalom, D.B. (2009) The medial prefrontal cortex and integration in autism. Neuroscientist 15, 589-598

103. Robertson, C.E. (2013) Slower rate of binocular rivalry in autism. J. Neurosci. 33, 16983-16991

104. Van Eylen, L. (2016) Executive functioning and local-global visual processing: candidate endophenotypes for autism spectrum disorder? In J Child Psychol. Psychiatry. Published online November 2, 2016. http://dx.doi.org/10.1111/jcpp.12637

105. Yatziv, T. and Jacobson, H. (2015) Understanding visual consciousness in autism spectrum disorders. Front. Hum. Neurosci 209, 204

106. Ameis, S.H. and Catani, M. (2015) Altered white matter connectivity as a neural substrate for social impairment in autism spectrum disorder. Cortex 62, 158-181

107. Ecker, C. et al. (2016) Relationship between cortical gyrification, white matter connectivity, and autism spectrum disorder. Cereb. Cortex 26, 3297-3309

108. Vasa, R.A. et al. (2016) The disrupted connectivity hypothesis of autism spectrum disorders: time for the next phase in research. Biol. Psychiatry Cogn. Neurosci. Neuroimaging 1 , 245-252

109. Dumas, G. (2012) Anatomical connectivity influences both intraand inter-brain synchronizations. PLoS One 7, e36414

110. Scott-Van Zeeland, A.A. (2010) Altered functional connectivity in frontal lobe circuits is associated with variation in the autism risk gene CNTNAP2. Sci. Transl. Med. 2, 56ra80

111. Changeux, J.P. and Christopoulos, A. (2016) Allosteric modulation as a unifying mechanism for receptor function and regulation. Cell 166, 1084-1102

112. Tsigelny, I.F. et al. (2015) Multiple spatially related pharmacophores define small molecule inhibitors of OLIG2 in glioblastoma. Oncotarget. Published online October 30, 2016. http:// dx.doi.org/10.18632/oncotarget.5633

113. Dehaene-Lambertz, G. and Spelke, E. (2015) The infancy of the human brain. Neuron. 88, 93-109

114. Mukherjee, P. and Owen, J. (2014) Tracking neurons' long projections to unravel autism. Spectrum 21 Octobe

115. Tsigelny, I.F. et al. (2016) Molecular mechanisms of OLIG2 transcription factor in brain cancer. Oncotarget. Published online July 16, 2016. http://dx.doi.org/10.18632/ oncotarget.10628 